\title{
Eddy currents in a passive magnetic axial thrust bearing for a flywheel energy storage system
}

\author{
Magnus Hedlund*, Johan Abrahamsson, Jesús José Pérez-Loya, Johan Lundin and \\ Hans Bernhoff \\ Division of Electricity, Department of Engineering Sciences, Uppsala University, Uppsala 75237, \\ Sweden
}

\begin{abstract}
Two types of passive magnetic lift bearings were evaluated in terms of thrust force and eddy current losses. The first type of bearings were based on two sets of segmented Halbach arrays mounted in repulsive mode, and the second type was based on ring-magnets. The eddy-currents studied arose in the bearing due to manufacturing variations of magnetic remanence, and due to non-radial magnetization. Both a 3D time-dependent and a quasi-stationary Finite-Element Method (FEM) formulation were used, and the simulated results were compared with lift-force measurements from experiment. The losses were found (by FEM) to be in the order of $25 \mathrm{~W}$ at a rotational speed of $30000 \mathrm{rpm}$ while lifting a $45 \mathrm{~kg}$ rotor with a stiffness of $359 \mathrm{~N} / \mathrm{mm}$.
\end{abstract}

Keywords: Flywheel energy storage, magnetic bearings, FEM, eddy-currents

\section{Introduction}

A kinetic energy storage for automotive applications is being developed at Uppsala University. Being an energy storage designed for many charge-discharge cycles with a high power to energy ratio, its place in a driveline is mainly as a short-term power buffer. Typical applications for this type of energy storage (described in depth in [7]) are power grid frequency regulation, uninterruptible power supply (UPS), and energy storage in vehicles. Recent examples of the latter include Formula 1 racing, and hybridization of London city buses. Also, the Audi car that won the 24-hour race in Le Mans for three consecutive years (2012-2014) was equipped with a flywheel.

The flywheel developed in Uppsala is part of a driveline, fully or partially electric depending on the main energy storage which may consist of a battery, a fuel cell or a diesel generator. A typical end application would be a city bus with frequent stops or a construction machine alternating idling periods with heavy work. The flywheel has many purposes, of which the most important is to protect the main energy storage from transients, thus increasing its lifetime and significantly lowering its power demands. In the case of the main energy storage being a battery, lowering the power demands will allow a higher

\footnotetext{
${ }^{*}$ Corresponding author: Magnus Hedlund, Division of Electricity, Department of Engineering Sciences, Uppsala University, Lägerhyddsvägen 1, Uppsala 75237, Sweden. Tel.: +46 184715804; E-mail: Magnus.Hedlund@ angstrom.uu.se.
}

1383-5416/17/\$35.00 (C) 2017 - IOS Press and the authors. All rights reserved

This article is published online with Open Access and distributed under the terms of the Creative Commons Attribution NonCommercial License (CC BY-NC 4.0). 


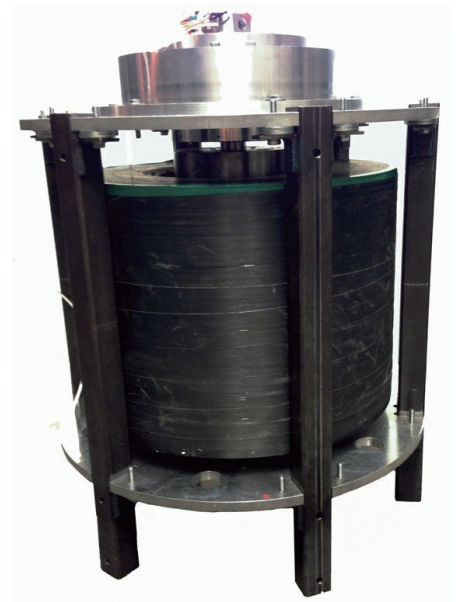

Fig. 1. Flywheel prototype currently being finalized at Uppsala University. The upper axial thrust bearing can be seen between the composite shell and the upper aluminum stator top cover, with the shaft passing through it.

energy density of the battery, thus decreasing the amount of batteries needed. The flywheel also enables efficient regenerative braking [8].

The rotor of the flywheel prototype as seen in Fig. 1 weighs $45 \mathrm{~kg}$ which gives a storage capacity of $800 \mathrm{Wh}$ at its maximum rotational speed of $30000 \mathrm{rpm}[2,3]$. It consists of a combination of a high-end carbon fiber and a less expensive glass fiber rim to lower production costs. To minimize stand-by losses, the flywheel rotor is operated in near vacuum and suspended on magnetic bearings. The bearings are divided into passive thrust bearings formed by segmented magnets operating in repulsive mode, and active electromagnets for radial positioning of the rotor. The passive thrust bearings are the focus of this paper.

Induced eddy currents can severly impact the functionality of electric machines, leading to decreased efficiency and power ratings, making it an important area of research. Specifically, bearing losses have been identified as an important factor in flywheel design [1], due to inherent poor cooling characteristics of a rotor suspended in vacuum. Heat sources on the rotor can affect fatigue limits of materials or worse, lead to overtemperature in the magnets resulting in demagnetization and delevitation. There are different ways of estimating eddy current losses, of which a few are listed here in rising order of computational complexity: Analytical solutions [5,15]; stationary Finite Element Method (FEM) solutions coupled with analytical equations [4,19]; quasi-stationary FEM-simulations [20]; and time-dependent FEM-simulations. Analytical equations have been developed to model electric machines with good accuracy compared to quasi-stationary 3D-FEM simulations [9]. Other recent interesting applications of the quasi-stationary 3D FEM-simulations for eddy currents include models of magnetic brakes [16] and in electromagnetical dampers [6]. An analytical model has been compared with the 3D time-dependent solution for an eddy current linear damping plate [11]. Solid pole electric machines have been analyzed with 2D time-dependent solutions [14]. Performance of 2D vs 3D FE eddy currents models for electric machines has also been under research [10].

This paper presents two FEM models for quantifying eddy current losses in two types of magnetic bearings (presented below) used in our flywheel setup. Our goal is to expand knowledge in this field by comparing the performance of the 3D quasi-stationary model to the full 3D time-dependent model for segmented magnetic bearings. As the losses are expected to be small and hard to distinguish from other losses in the system, the models are an effective alternative to building an expensive measurement setup. 


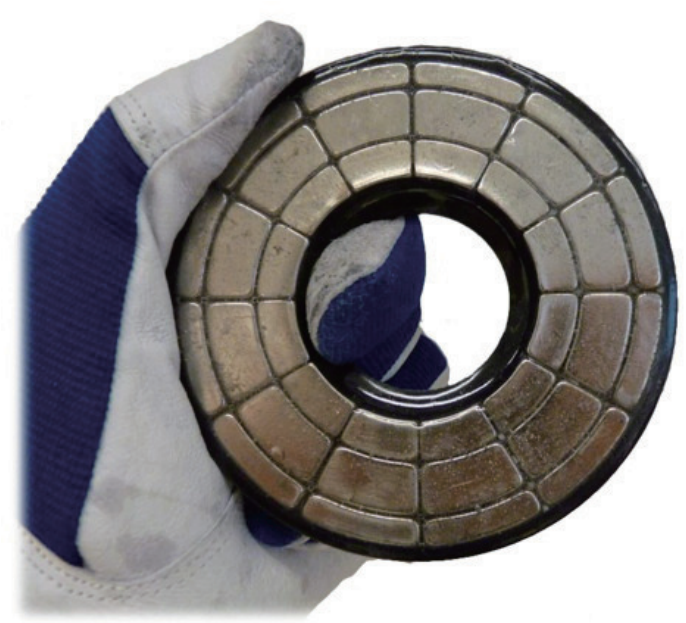

Fig. 2. A segmented unit. It constitutes one half of the segmented Halbach bearing. The inner row is magnetized inwards, the middle row radially outwards and the outer row outwards.

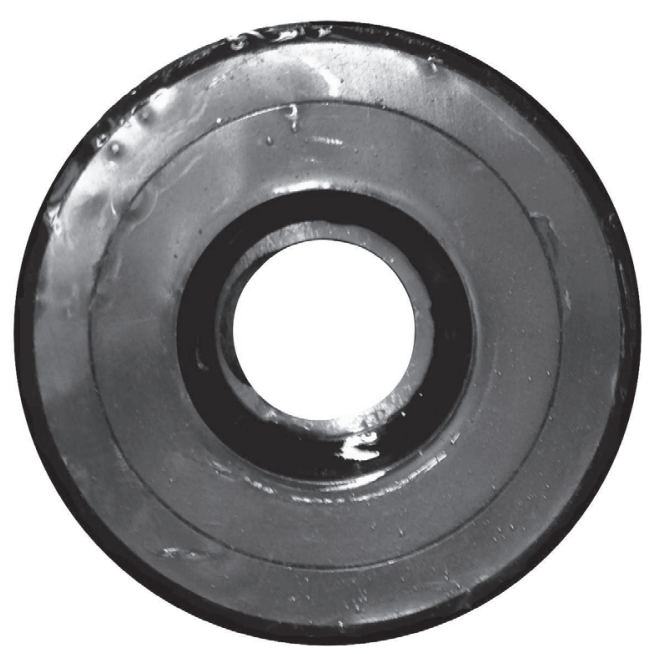

Fig. 3. A ring-magnet unit, which constitutes one half of the semi-segmented bearing, with magnetic fields directed inwards for the inner magnet. The opposite applies for the outer magnet.

It also enables geometrical optimization of future designs. The simulation models were constructed and validated against experimental data of lift force. It is shown that the full solution is necessary to see the benefits of the segmented bearing design. Two sources of inhomogenities in the magnetic field are studied (non-radial magnetization and magnetic remanence variations). The study is limited to perfectly aligned rotors. Finally, the system performance is evaluated for the rotor suspension system (both upper and lower axial bearings).

\subsection{Magnetic axial thrust bearings}

The magnetic axial thrust bearing under study is passive, comprised of magnets operating in repulsive mode. Two units, one mounted on the rotor and one in the stator, make up a bearing configuration. Two different types of units are considered, in two different configurations, as described below.

\subsubsection{Bearing units}

Segmented units

The segmented unit (SU) consists of three rows of magnets of different magnetizations, separated circumferentially into 12 different radial columns, as seen in Fig. 2. The magnets are ordered radially in a Halbach array, which concentrates the magnetic field above the unit [21]. The magnets are resting in a plastic cup, fastened with epoxy. The plastic cup itself rests inside a high-strength aluminum cup for mechanical stability. The non-conductive plastic cup serves to create some extra distance between external varying magnetic fields and the aluminum support structure. A similar unit was under study in a different setting in [12].

The Halbach matrix is hard to assemble, but the benefit of not using back-iron to complete the magnetic circuit contributes to lower the eddy current related losses since the main flux path does not permeate any highly conductive materials. Any propagated variations in flux due to imperfections will only travel through air, plastic and magnets. Also, the units can sustain high centrifugal loads since the magnets are free to expand radially. 


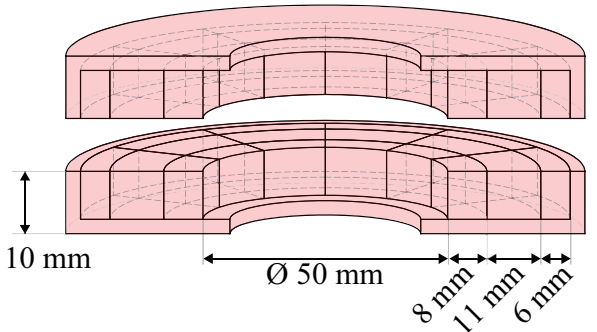

(a) FSC bearing, with two red SUs facing each other

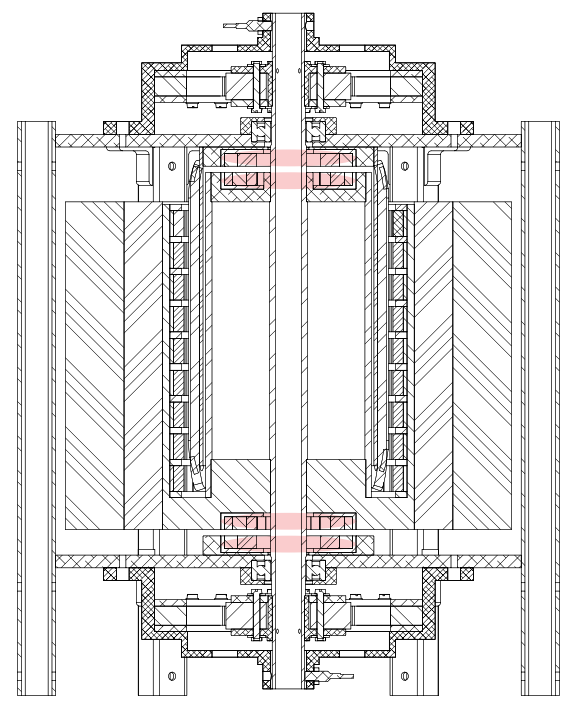

(c) FSC bearings mounted in the flywheel

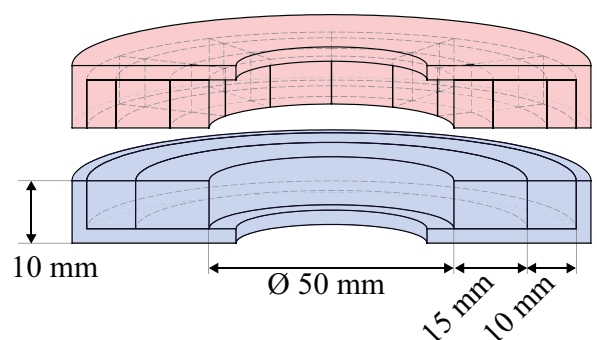

(b) SSC bearing, with a blue RMU facing an SU

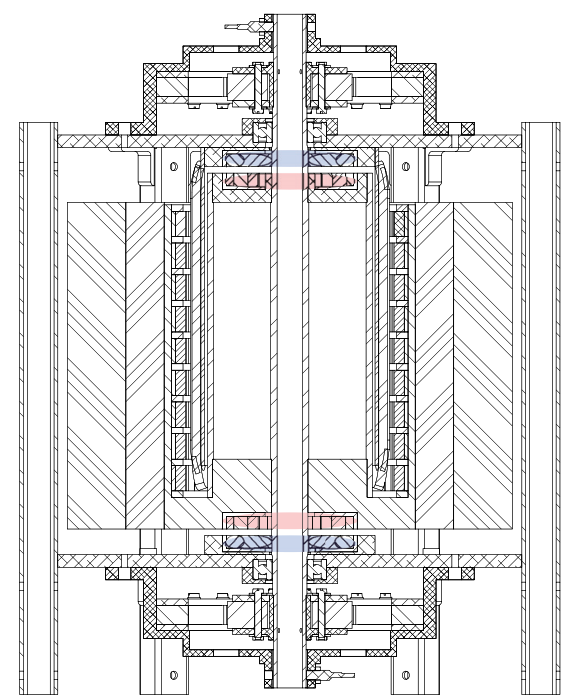

(d) SSC bearings mounted in the flywheel

Fig. 4. Schematics showing different configurations, where $4 \mathrm{a}$ and $4 \mathrm{~b}$ are studied with FEM and in experiment. The results are then used to estimate system performance when these configurations are mounted as shown in $4 \mathrm{c}$ and $4 \mathrm{~d}$. The unit types are color coded, where red and blue corresponds to SUs and RMUs respectively.

Assuming that the two units are perfectly aligned around the same rotational axis, variations in the magnetic field around the circumference arise due to the following reasons:

- Non-radial magnetization: The radial magnets required to form a Halbach array cannot be magnetized truly radially during manufacturing.

- Remanence variation: The remanence of each individual magnet might vary from batch to batch.

- Small geometrical variations: Depends on the tolerances in the production process. Not studied here.

\section{Ring-magnet units}

The ring-magnet unit (RMU) consist of two rings magnetized axially (up and down, respectively), seen in Fig. 3. A similar structure for radial bearings can be seen in [18]. The magnets do not form a Halbach array, but they help concentrate the flux, producing a slightly concentrated radial return path through the air gap. The radial ring magnet required to form a Halbach array is not available due to practical limitations in the manufacturing process. The flux distributes equally above and below the unit, creating a lot of leakage flux below the unit, at the expense of lift force. However, the leakage flux does 
not contribute directly to losses, since it is rotationally symmetric from the point of an observer on the rotor.

Since NdFeB is very brittle, these units become very sensitive to centrifugally induced radial expansion on the rotor. For example, the ultimate tensile strain (UTS) of NdFeB magnets is only $0.05 \%$, while modern composite fibers can have UTSs larger than $2 \%$. If these units were mounted on the rotor (at some not very small fraction of the maximal rotor radius) they could impose a serious limitation to the maximal rotational speed. Therefore, these units are only studied for placement in the stator.

The benefits of these units include:

- The flux they produce is rotationally symmetric, even if the magnetization strength varies from batch to batch.

- There is no need for a radial magnetization, which completely removes the effect of non-radial field.

\subsubsection{Bearing configurations}

Fully segmented configuration

Two SUs make up one FSC-bearing (Fully Segmented Configuration), as seen in Fig. 4. An investigation of the lift-force and a simple eddy-current analysis of this bearing were presented in [2]. Since both units are Halbach arrays, the force per unit volume is high. However, there are induced losses in both the upper and lower unit, due to natural variations in the magnetic field from the SU-type. Losses in the rotor can be a significant drawback for vacuum operation, since cooling options are limited. In the particular flywheel device studied, the whole rotor is levitated on magnets, and the only cooling mechanism is then radiation.

\section{Semi-segmented configuration}

An SSC-bearing consists of the SU mounted co-axially towards an RMU. Therefore, another spinning unit placed on the symmetric axis of the RMU will experience no induced eddy-currents. Having one of these units mounted on the stator will thus decrease the amount of produced heat in the difficult-to-cool flywheel rotor.

\subsubsection{Inter-magnet insulation}

All the individual magnets mentioned above are covered in a thin layer of protective $\mathrm{Ni} \& \mathrm{Cu}$-coating, which is conductive. The eddy currents can be limited in magnitude by isolating the magnets in the segmented units with an additional coating of varnish, which can be added during manufacturing. The implications of insulation can be taken into account in the simulations, as seen in the results below. However, since varnish can decrease heat conductivity, there may be implications to bearing cooling.

\section{Method}

The commercial FEM-solver COMSOL Multiphysics was used to evaluate lift force and eddy current losses in the magnetic bearing.

\subsection{Potential equations}

The solver equations on which FEM is applied are derived from Maxwell's equations with all relevant assumptions here. The well-known Ampère's law (relates the magnetic field $\mathbf{H}$, the free current 
distribution $\mathbf{J}_{f}$ and the electrical displacement field $\mathbf{D}$ ), Faraday's law (relates the electric field $\mathbf{E}$ and the magnetic flux density $\mathbf{B}$ ) and the magnetic potential definition (denoted $\mathbf{A}$ ) are:

$$
\begin{aligned}
& \nabla \times \mathbf{H}=\mathbf{J}_{f}+\frac{\partial \mathbf{D}}{\partial t} \\
& \nabla \times \mathbf{E}=-\frac{\partial \mathbf{B}}{\partial t} \\
& \mathbf{B}=\nabla \times \mathbf{A}
\end{aligned}
$$

The electric field is assumed to be generated only by magnetic induction $(\nabla \cdot \mathbf{E}=0)$.

Equations 2 and 3 combines into:

$$
\nabla \times\left(\mathbf{E}+\frac{\partial \mathbf{A}}{\partial t}\right)=0
$$

or, equivalently:

$$
\mathbf{E}=-\frac{\partial \mathbf{A}}{\partial t}
$$

The magnetic material model is a first order model based on magnetic remanence $\mathbf{B}_{r}$ and relative magnetic permeability $\mu_{r}$.

$$
\mathbf{B}=\mu_{0} \mu_{r} \mathbf{H}+\mathbf{B}_{r}
$$

Given a moving observer (denoted with prime) of velocity $\mathbf{v}$, a stationary magnetic field will induce an apparent electric field in the moving frame [17]:

$$
\mathbf{E}^{\prime}=\mathbf{E}+\mathbf{v} \times \mathbf{B}
$$

Assuming no other external sources of current, the free current density can be rewritten as (with conductivity $\sigma$ ):

$$
\mathbf{J}_{f}=\mathbf{J}^{\prime}=\sigma \mathbf{E}^{\prime}=\sigma(\mathbf{E}+\mathbf{v} \times \mathbf{B})
$$

\subsubsection{Full potential}

When solving for the full potential, it is assumed that $\mathbf{B}_{r}=0$ and $\sigma \neq 0$. The displacement current is also assumed to be negligible for the given frequency ranges. A potential equation can thus be written as a combination of Eqs 1, 3, 6 and 8.

$$
\nabla \times\left(\frac{\nabla \times \mathbf{A}}{\mu_{0} \mu_{r}}\right)+\sigma \frac{\partial \mathbf{A}}{\partial t}-\sigma \mathbf{v} \times(\nabla \times \mathbf{A})=0
$$

The full potential described by Eq. (9) is used to model moving conductive domains in the timedomain exposed to an externally generated magnetic field.

\subsubsection{Scalar potential}

In contrast to the assumptions for the full potential equation, here it is assumed that $\mathbf{B}_{r} \neq 0$ and $\sigma=0$. Since the only free currents assumed to be present were magnetically induced (from Eq. (8)), the conductivity assumption above reduces Ampère's law to its rotation-free form:

$$
\nabla \times \mathbf{H}=\mathbf{J}_{f}=0
$$

which enables the use of a scalar potential $\phi_{m}$ (by applying Helmholtz's Theorem):

$$
\mathbf{H}=-\nabla \phi_{m}
$$




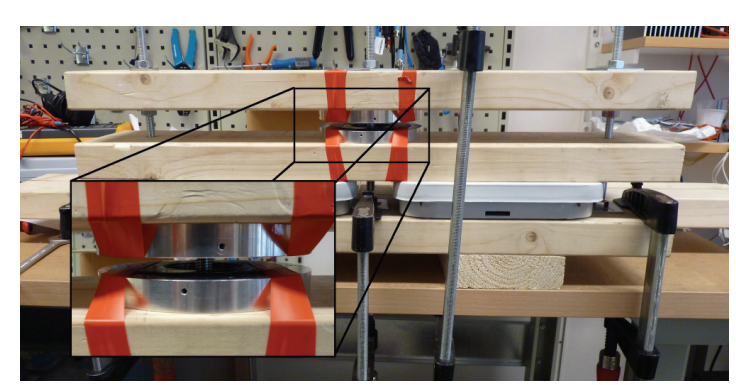

Fig. 5. Test-bench used for axial force measurement, seen here in a partially dissassembled state.

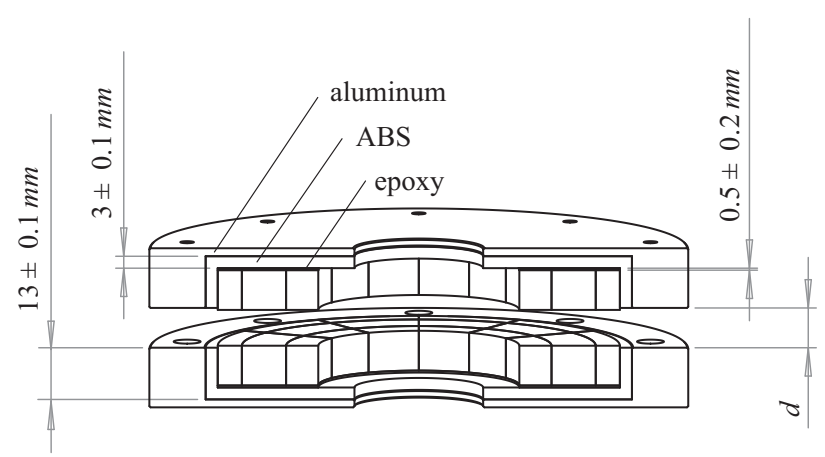

Fig. 6. Schematic view of the measurement setup with the relevant dimension tolerances (all given in $\mathrm{mm}$ ). The distance $d$ between the aluminum cups is used to compute the airgap, but manufacturing tolerances as well as a layer of epoxy applied during assembly adds uncertainty to the measurement. Not shown is the height tolerances of the magnets themselves, which is $10.0 \pm$ $0.1 \mathrm{~mm}$.

By inserting Eqs. 6 and 11 into Gauss' law for magnetism, a simpler equation is attained:

$$
\nabla \cdot\left(\mathbf{B}_{r}-\mu_{0} \mu_{r} \nabla \phi_{m}\right)=0
$$

The velocity term is rewritten to reflect (perfectly aligned) rotary motion in a cylindrical system, as follows $(r, \varphi, z)$ :

$$
\mathbf{v}=2 \pi r f \hat{\varphi}
$$

where $f$ is the rotational frequency of the bearing.

\subsection{Lift force}

\subsubsection{Simulation}

The (stationary) scalar magnetic potential (Eq. (12)) was solved for the lift force simulation, and the Maxwell stress tensor was used to compute the force. The manufacturing uncertainty in the magnetization was taken into account by evaluating the magnetization interval end points, creating a lift-force uncertainty band.

\subsubsection{Experimental measurement}

Two units were mounted in a stationary setup for evaluation of standstill lift-force, as seen in Fig. 5. The bearings were centered above each other and compressed axially. The axial repulsive force was then measured for both the FSC and SSC respectively.

The airgap was indirectly measured as the quantity $d$ in Fig. 6, and then computed via geometry. A few inaccuracies arose due to this approach, specifically:

- Manufacturing tolerances (each $\pm 0.1 \mathrm{~mm}$ ) in the machining tools for the:

* aluminum outer cup

$*$ plastic inner cup (ABS)

$* \mathrm{NdFeB}$ magnets

- Uncertainty in the thickness of epoxy applied when glueing the magnets into a halbach configuration, measured to be $0.5 \pm 0.2 \mathrm{~mm}$. 


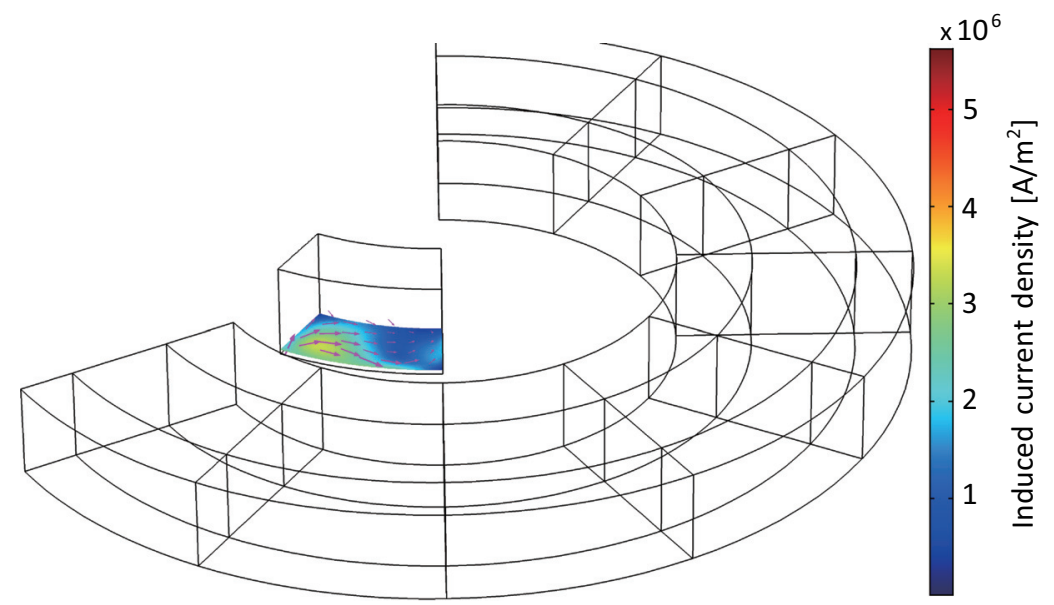

Fig. 7. Geometry for the FSC 3D time-dependent simulation. One conductive magnet-triplet (in blue) is flying past magnet-triplets below. The lower magnets have a remanent magnetization, but are non-conductive. The upper magnet-triplet is conductive, but with no remanent magnetization. The magnets in the triplet are simulated individually.

These uncertainties were assumed to behave uniformly, resulting in a worst case accuracy (due to measurement recomputation) of $\pm 0.5 \mathrm{~mm}$.

The uncertainties in caliper and weight scale were considered small in comparison to the above.

\subsection{Eddy current simulation}

\subsubsection{Time-dependent solution}

A time-dependent magnetic simulation of the bearing was performed, to account for both of the last terms in Eq. (9). All non-conductive domains were solved for the scalar potential, and the conductive magnets were solved for the full potential. Three radially distributed magnets $\left(\mathbf{B}_{r}=0\right.$ and $\left.\sigma \neq 0\right)$ were moving above a total of 9 of magnets $\left(\mathbf{B}_{r} \neq 0\right.$ and $\left.\sigma=0\right)$, as seen in Fig. 7.

To avoid time-consuming remeshing every time the geometry is updated, the whole mesh of the upper air cylinder was rotated along with the moving magnets. Since the mesh nodes on the boundary between the rotating and stationary air domains (generally) do not coincide, the equations were connected via an interpolated boundary. By using the relatively simple scalar potential on both sides of the interpolation boundary, additional computational effort was kept to a minimum. An increase in mesh density of about a factor 3 on the stationary source boundary was applied, since it was found to improve the solver convergence rate.

The boundaries between the full and scalar potential boundaries were coupled with a COMSOL provided condition named Mixed Formulation Boundaries, connecting the different differential equations.

The induced currents will appear in the conductive domain, so the losses for one bearing configuration is a combination of two simulations; currents induced by the rotating unit inside the stationary and the opposite. The losses are thus defined from a unit (onto another unit).

\subsubsection{Loss computation}

The total eddy current losses were computed as an integral over one revolution of momentary loss. The momentary losses were computed as [17]:

$$
P_{\text {loss,momentary }}(t)=\int_{\text {Volume }} \mathbf{J}_{f}(t) \cdot \mathbf{E}(t) d V
$$


which in turn yields to the total loss expression, integrated over one revolution, and multiplied with the frequency:

$$
P_{\text {loss }}=f \int_{t=0}^{t=1 / f} P_{\text {loss }, \text { momentary }}(t) d t
$$

\subsubsection{Quasi-stationary}

A quasi-stationary approximation of the time-dependent solution is attained if $\frac{d \mathbf{A}}{d t}$ is neglected, which, if applied to Eq. (9), yields an equation devoid of $t$ which can be readily solved with a stationary solver:

$$
\nabla \times\left(\frac{\nabla \times \mathbf{A}}{\mu_{0} \mu_{r}}\right)-\sigma \mathbf{v} \times(\nabla \times \mathbf{A})=0
$$

The approximation above is valid when geometry, material properties and magnetic source distribution (divergence of the magnetization, $\nabla \cdot \mathbf{M}$ ) of the rotating part are rotationally symmetric. In the case of the SUs in which the magnets are electrically isolated from each other, the above conditions do not apply, resulting in a degradation of accuracy.

\subsubsection{Field variation boundary conditions}

Magnetic field variations from two sources are under study, as mentioned in Section 1.1. The two effects are studied separately.

\section{Remanence variations}

The material data used in the simulations was given by the manufacturer, who claims the remanence to be in the interval of 1.35 and $1.40 \mathrm{~T}$ for the particular $\mathrm{N} 48$-grade $\mathrm{NdFeB}$ magnets used. A worst-case scenario was studied, where every other magnet around the circumference was assumed to be at opposite ends of the remanence interval.

\section{Non-radial magnetization}

The non-radial magnetization effect applies only to the middle magnets of the SUs, as discussed earlier. Ideally, the magnetic remanence is perfectly radial, implying that every mesh point inside the magnet domain has a slightly different remanence direction. However, the magnetization process only allows for a parallel magnetization. A Cartesian coordinate system is thus used to define the magnetization inside the magnet domains, producing parallel remanence in every mesh point.

\subsubsection{Electrical insulation of magnets}

The internal conductivity of the NdFeB magnets was assumed to be $6.9444 \mathrm{M} \frac{\mathrm{S}}{\mathrm{m}}$ and the relative magnetic permeability was assumed to be 1.05 . The magnets are coated in a thin protective film, whose conductivity was neglected in this study. Instead, two extreme cases were studied:

- Isolated: Zero conductivity between adjacent magnets.

- Non-isolated: Internal conductivity applied to the boundaries of the magnet.

Note that for a given magnetic field source unit in the non-isolated case, the moving, conductive, zeroremanence unit can be modeled without interior boundaries since the solution for the SU and RMU will be the same.

\subsection{System losses and stiffness}

The rotor is held in place by two sets of axial bearings, mounted above and below the rotor. 


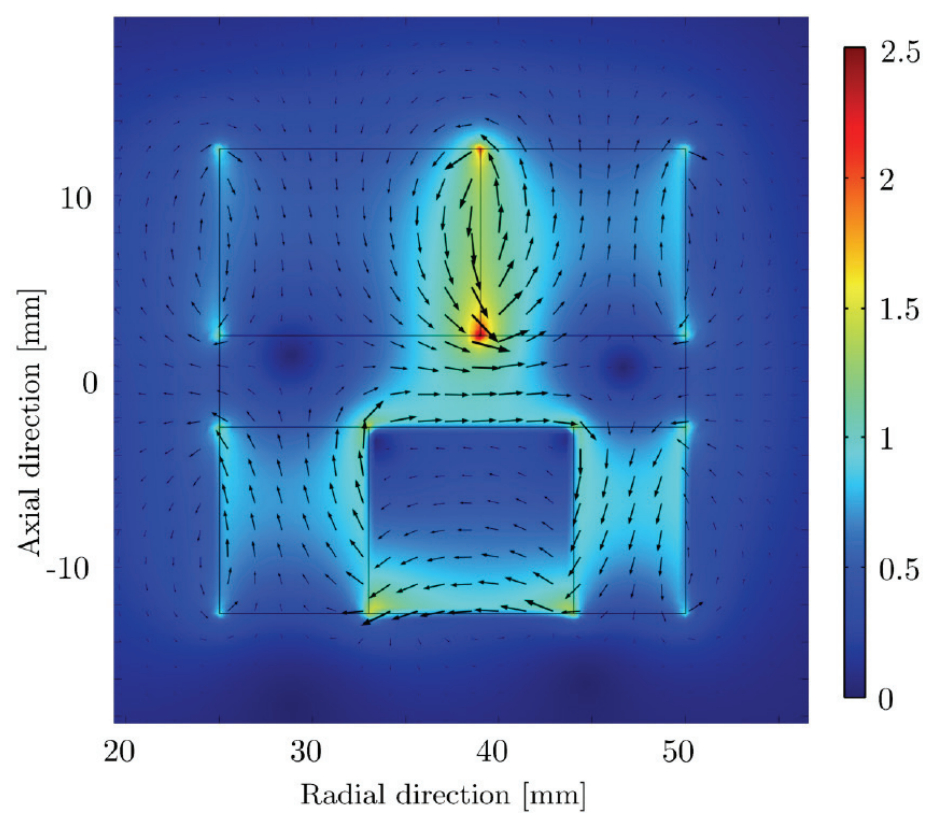

Fig. 8. A radial-axial cutaway view of the magnetic flux density (in T) at an air gap of $5 \mathrm{~mm}$ of an SSC bearing with an RMU on top and an SU in the bottom.

Gravitational forces offsets the rotor by a distance $d$ until the bearings collectively lifts $45 \mathrm{~kg}$. Denote the nominal airgap $a_{\text {nom }}$, then the axial forces $F$ in standstill sum up to

$$
F_{\text {bearing, upper }}+m g-F_{\text {bearing, lower }}=F\left(a_{\text {nom }}+d\right)+m g-F\left(a_{\text {nom }}-d\right)=0 .
$$

The force in relation to airgap can be used to solve Eq. (17) for $d$. The total stiffness and losses can then be computed for the full system (rotor and both upper and lower bearings). The axial stiffness is defined as the derivative of the total axial force with respect to $d$.

\section{Results}

\subsection{Lift force}

A 2D cut-through of the magnetic flux density from the FEM-simulation can be seen in Fig. 8. Measurements and results from simulations are plotted for both bearing configurations in Fig. 9. With the uncertainty assumptions presented earlier, corresponding error margins are seen as shaded areas around the experimental data points.

The simulation was performed for the interval endpoints of the magnetic remanence, depicted as a shaded area in Fig. 9.

The SSC bearing features about three fourths of the lift-force relative to the FSC.

\subsection{Quasi-stationary eddy-current simulation}

\subsubsection{Non-radial magnetization}

The losses due to non-radial magnetization were computed for both FSC and SSC configurations, as seen in Fig. 10. The rotational speed was $500 \mathrm{~Hz}$, which is full speed for the flywheel prototype presented above. The losses vary with angular position for the FSC, as seen in Fig. 12. 


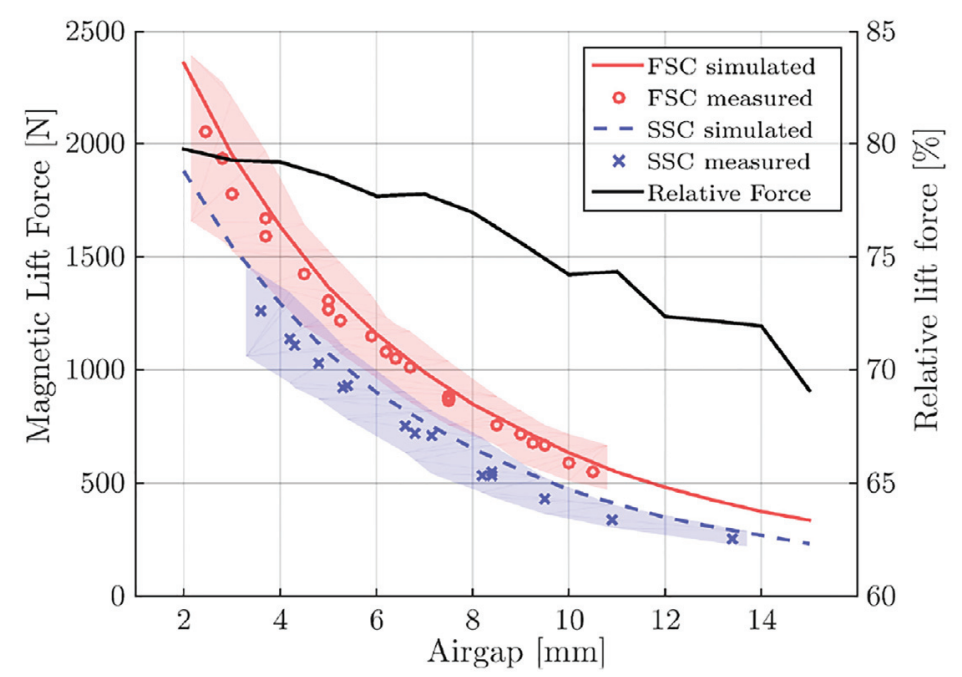

Fig. 9. Lift force as a function of air gap size for the FSC and the SSC bearings. The measurements of the SSC correspond well to the theoretical simulations (the FSC curve was validated experimentally in [2]). It is seen that a semi-segmented bearing features about $75 \%$ relative lift force compared to the fully segmented concept.

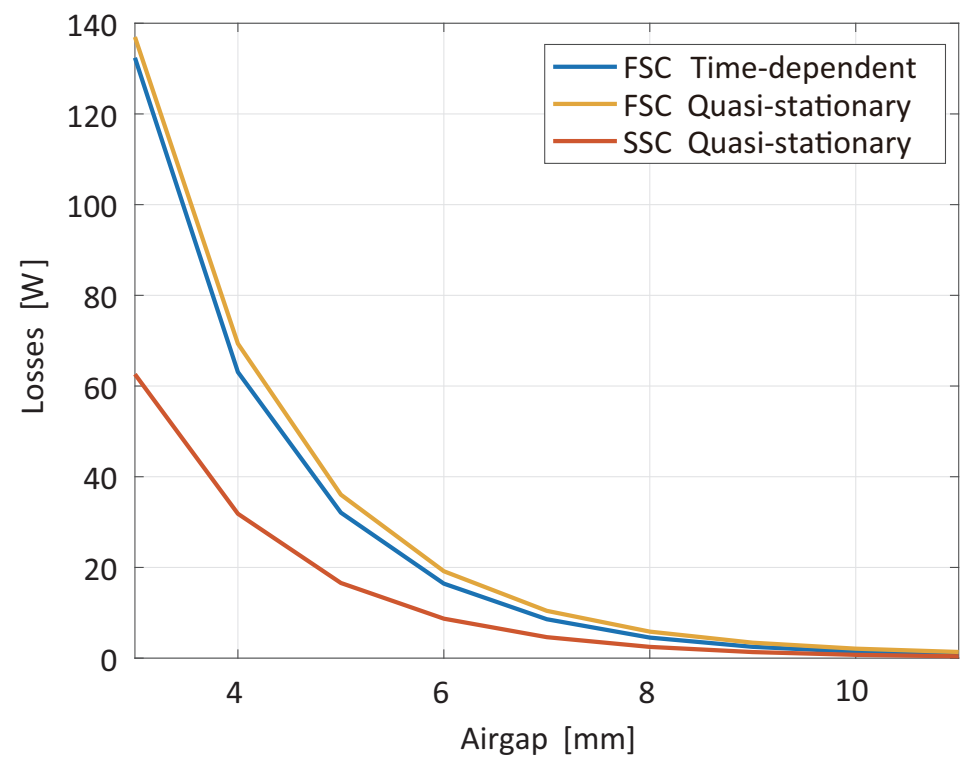

Fig. 10. Bearing losses as a function of airgap. These losses were produced by non-radial magnetization effects.

\subsubsection{Remanence variation}

As seen in Fig. 11, the losses due to remanence variations are substantially lower than those associated with a non-radial magnetization. Half of the magnets were magnetized to $1.35 \mathrm{~T}$, and the other (radially distributed) triplets of magnets were all magnetized to some value between $1.35 \mathrm{~T}$ and $1.40 \mathrm{~T}$. When traversing the circumference, each variation in magnetization results in some losses (the simulation in the figure is a result of one of these transitions). The resulting bearing loss is a combination of every transition, and is thus dependent on some remanence distribution function. A worst case scenario was 


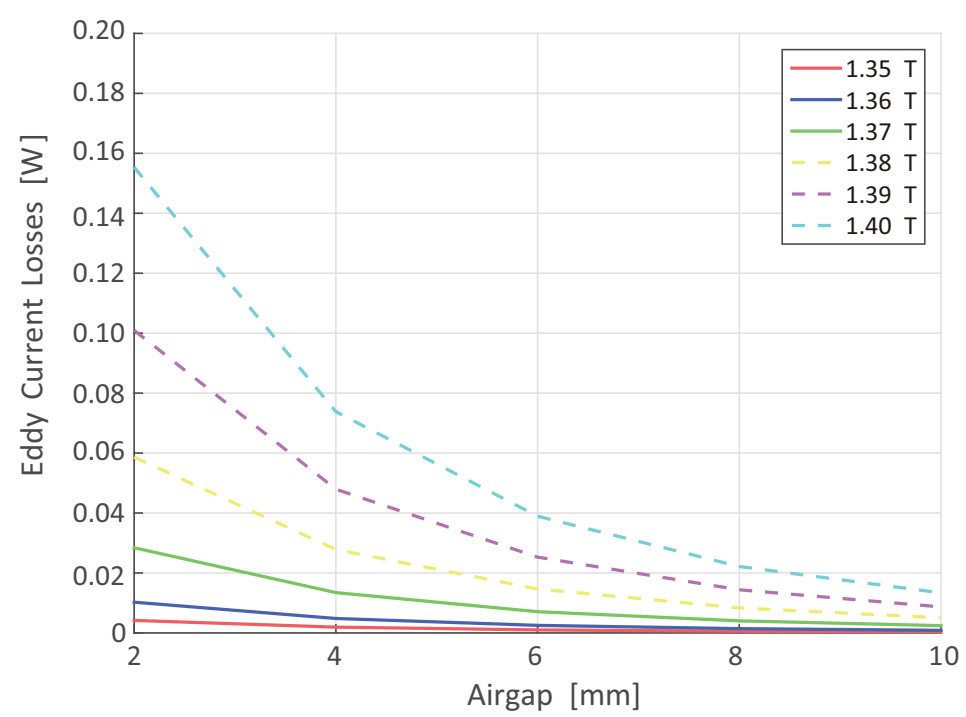

Fig. 11. Losses in non-isolated bearing due to one magnetic triplet in an SU having a different remanence (a single transition), as a function of air gap and remanence deviation. Every other magnet was magnetized to $1.35 \mathrm{~T}$. By combining transitions around the circumference, the worst-case scenario losses at $6 \mathrm{~mm}$ air gap became $0.48 \mathrm{~W}$.

created (assuming the remanence is confined to an interval $B_{r} \in[1.35,1.40]$ by letting every other magnet triplet be the minimum and the maximum respectively. The worst case $\Delta B_{r}$-losses for one segmented unit at an air gap of $6 \mathrm{~mm}$ at $500 \mathrm{~Hz}$ was $0.48 \mathrm{~W}$.

\subsection{Time-dependent eddy-current simulation}

The corresponding time-dependent solutions for the losses related to non-radial magnetization were lower than for the quasi-stationary case. Figures 10 and 12 shows losses for an FSC bearing in timedependent simulations.

\subsection{System stiffness and losses}

The end application defines what frequencies will be exciting the rotor axially. In turn, the rotor mass and total axial stiffness defines the natural frequency (of a rigid rotor). The system total stiffness given a certain nominal airgap can be seen in Fig. 13, along with the system losses.

\section{Discussion}

\subsection{Lift force}

Since half the flux from the RMU escapes as leakage flux (compared to the SU, which has almost no leakage flux in comparison), a decrease in thrust force was expected and verified.

The coefficient of determination when applying an exponential model $\left(y=k_{1} e^{k_{2} x}\right)$ was 0.9954 and 0.9971 for the FSC and SSC respectively. This suggests that the spread of the measurements are low. However, the mean relative difference between the exponential model and the FEM simulations are 10 and $16 \%$ for the FSC and SSC, suggesting some systematic measurement error or other unmodeled effect. 


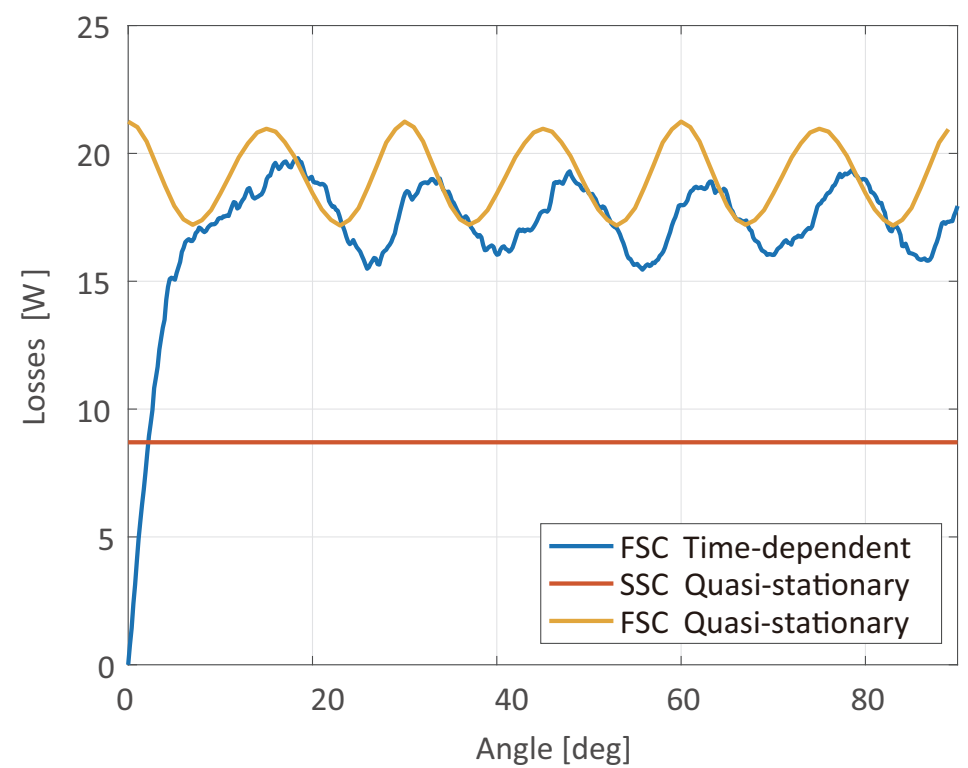

Fig. 12. Bearing losses as a function of angular position, as computed by quasi-stationary methods for both configurations, and by time-dependent methods for the FSC.

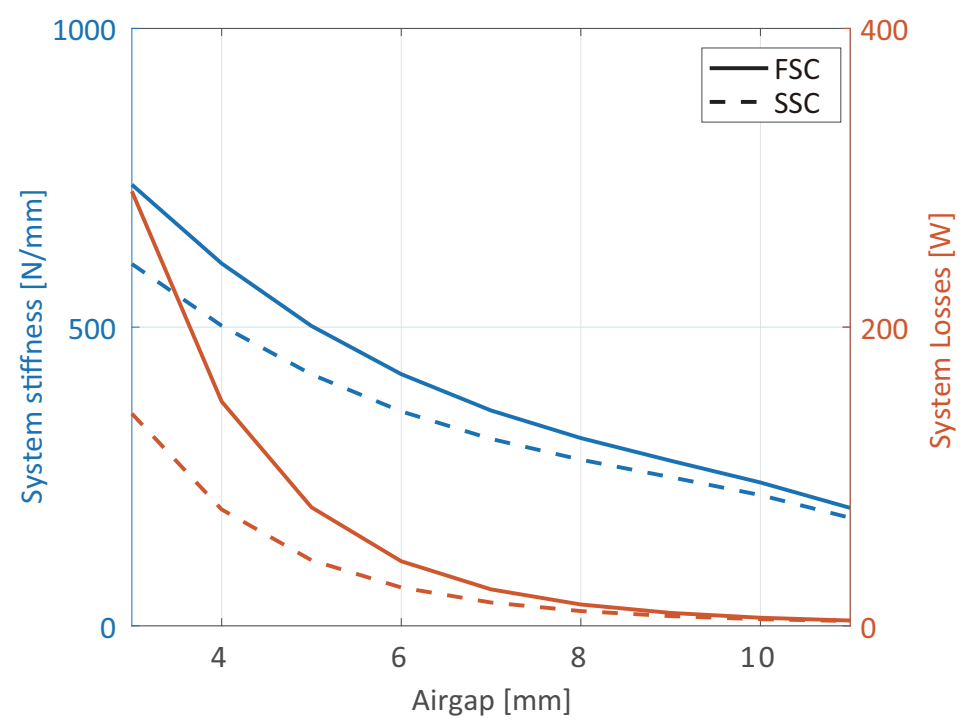

Fig. 13. System performance when two bearings are mounted in the flywheel system. The system thrust force is $441 \mathrm{~N}$. The airgap on the $\mathrm{x}$-axis is the nominal airgap. Note that the losses for the SSC at similar or better stiffness.

\subsection{Eddy current losses}

In Fig. 10 it can be seen that for the heavily segmented FSC bearing, the losses are significantly lower (about 10\%) in the time-dependent simulation than the quasi-stationary. The eddy-currents in the timedependent solution was seen to have a settling time of about 30 degrees (or about $166 \mu$ s), probably driven by a self-inductive behaviour. 
Note that the mesh for the time-dependent formulation is coarser, due to a higher computational demand.

The frequency dependent losses due to non-radial magnetization exhibited inverse exponential behaviour $\left(R^{2}=0.9999\right)$ with increasing airgap, and quadratic behaviour $\left(R^{2}=1.0000\right)$ with increasing frequency. Similar results were obtained for the losses due to remanence variations seen in Fig. 11, with a verified quadratic behaviour with increasing field strength.

The dominant loss effect seems to be due to non-radial magnetization. The remanence variation losses is seen to be at least two orders of magnitude smaller (in a worst case scenario). The requirements on the magnet manufacturer to reduce remanence variations can thus be less strict.

\subsection{Simplifications}

As presented in the method, the moving unit is not magnetized, so the only field present is; from the source (standstill) unit, and from the induced eddy-currents in the moving unit. The eddy-currents will thus decrease the field strength, thus decreasing bearing capacity (stiffness). Another type of simulation with both units magnetized and conductive would have to be performed to see if these effects would be enhanced.

Isolated magnets are simulated one by one, the influence from eddy currents in magnet to its neighbour is thus not modeled here.

In [2], the magnitude of the losses are estimated by the stationary velocity term described above, although at only one angle. Also, the conductivity used in this article is half the value used there.

\subsection{Computational complexity}

The time-dependent solution was found to be quite computationally demanding compared to the stationary loss-simulation due to the need of many intermediate time-steps. The stationary solution could be computed over just the relevant angles. However, the time-depdendent solution is more sensitive to the geometry of the bearings, and it can be seen that the losses in the SSC bearing is estimated to be 25\% lower than for the quasi-stationary approximation.

\subsection{Meshing of quasi-stationary remanence variation}

The losses due to remanence variations were found to be small, so the meshing had to be done with care to distinguish the effect from computational artifacts.

A tetrahedral mesh did not produce any usable results at all, the solution contained unwanted induced currents in locations with a uniform magnetic field even though a high number of mesh elements were used. The ratio of induced currents to mesh artifacts was relatively poor, only 5:1. A hexahedral mesh produced much less of these mesh-induced currents for the same amount of elements, but artifacts were still visible. Further mesh refinement led to an induced currents to artifacts ratio of 50:1, which was found to be an acceptable trade-off between computational time and accuracy. The number of mesh elements were tripled during this process.

Note that these artifacts only appeared when applying a radial magnetization to a domain, the nonradial magnetization solution above did not suffer from these artifacts. The artifacts are probably an effect of discrete magnetization in single mesh points, causing a variation around the circumference which is suppressed when increasing total number of mesh points. 


\subsection{Time-dependent remanence variations}

The remanence variations were too small to distinguish from computational noise by time-dependent simulation, due to requirements on the mesh as discussed above. A test case with a larger step in remanence (from 0 to $1.35 \mathrm{~T}$ ) was used to compare how the time-dependent and quasi-stationary methods handled variations in magnetization intensity. These two were found to produce similar results.

\section{Conclusion}

An eddy current loss model was created and partly validated by comparing numerical model with lift force measurements. The presented numerical model predicted small losses (which are hard to measure without expensive experimental setups). The results and the tool obtained are useful for future thermal simulations or geometrical optimizations.

The SSC-bearing has a stiffness of $359 \mathrm{~N} / \mathrm{mm}$ at an airgap of $6 \mathrm{~mm}$, with losses at $500 \mathrm{~Hz}$ of about $25 \mathrm{~W}$, while lifting the rotor of $45 \mathrm{~kg}$. The SSC had its losses in the stator. The FSC-bearing has a stiffness of $421 \mathrm{~N} / \mathrm{mm}$, with losses of about $43 \mathrm{~W}$, spread evenly between rotor and stator. The SSC and FSC bearings thus produce losses per unit stiffness of $69 \mathrm{Wm} / \mathrm{N}$ and $102 \mathrm{Wm} / \mathrm{N}$ respectively, suggesting that the SSC is $32 \%$ more efficient even though it produces less thrust per volume.

Two eddy current sources were studied, firstly induction due to non-radial magnetization of the middle magnets in the SUs, and secondly induction due to manufacturing variations in magnetic remanence. These two effects were isolated into different simulations, and the non-radial magnetization was estimated to induce more than 10x losses than remanence variations.

The fully segmented bearing had lower losses when simulated with time-dependency, due to selfinductive effects in the eddy-currents. The time-dependent thus capture the dynamics of non-rotationally symmetric objects better.

\section{Future work}

The assumption of perfect centering could be loosened to include gyroscopic effects as seen in [5]. However, this increases the complexity of the FEM problem since a larger part of the geometry (if not full) must be included if the shaft is rotating off center. As seen in recent publications, the radial orbits for a passive magnetic bearing can vary greatly with rotational speed [13].

\section{Acknowledgments}

This work was conducted within the STandUP for Energy strategic research framework, and supported by The Swedish Energy Agency.

\section{References}

[1] J. Abrahamsson, J.G. de Oliveira, J. de Santiago, J. Lundin and H.Bernhoff, On the Efficiency of a Two-Power-Level Flywheel-Based All-Electric Driveline, Energies 5(12) (August 2012), 2794-2817.

[2] J. Abrahamsson, M. Hedlund and H. Bernhoff, Kinetic Energy Storage for Vehicular Applications, In 13th International Symposium on Magnetic Bearings, Virginia, USA, 2012. 
[3] J. Abrahamsson, M. Hedlund, T. Kamf and H. Bernhoff, High-Speed Kinetic Energy Buffer: Optimization of Composite Shell and Magnetic Bearings, IEEE Transactions on Industrial Electronics 61(6) (June 2014), 3012-3021.

[4] N. Boubaker, D. Matt, P. Enrici, T. Martire and F. Nierlich, Estimation of rotor eddy-current losses for high speed SPMSM, In 2013 15th European Conference on Power Electronics and Applications (EPE), September 2013, 1-6.

[5] O. Bouty, Eddy current losses in passive magnetic bearings, Journal of Applied Physics 92(11) (2002), 6851-6856.

[6] J.G. Detoni, Q. Cui, N. Amati and A. Tonoli, Modeling and evaluation of damping coefficient of eddy current dampers in rotordynamic applications, Journal of Sound Vibration 373 (July 2016), 52-65.

[7] M. Hedlund, J. Lundin, J. de Santiago, J. Abrahamsson and H. Bernhoff, Flywheel Energy Storage for Automotive Applications, Energies 8(10) (September 2015), 10636-10663.

[8] J. Lundin, Flywheel in an all-electric propulsion system. Licenciate Thesis, Uppsala Universitet, Uppsala, Sweden, 2011.

[9] M. Mirzaei, A. Binder, B. Funieru and M. Susic, Analytical Calculations of Induced Eddy Currents Losses in the Magnets of Surface Mounted PM Machines With Consideration of Circumferential and Axial Segmentation Effects, IEEE Transactions on Magnetics 48(12) (December 2012), 4831-4841.

[10] S. Niu, S.L. Ho, W.N. Fu and J. Zhu, Eddy Current Reduction in High-Speed Machines and Eddy Current Loss Analysis With Multislice Time-Stepping Finite-Element Method, IEEE Transactions on Magnetics 48(2) (February 2012), 10071010.

[11] K.J.W. Pluk, T.A. van Beek, J.W. Jansen and E.A. Lomonova, Modeling and Measurements on a Finite Rectangular Conducting Plate in an Eddy Current Damper, IEEE Transactions on Industrial Electronics 61(8) (August 2014), 40614072.

[12] E. Rodriguez, J. de Santiago, J.J. Pérez-Loya, F.S. Costa, G.G. Sotelo, J.G. Oliveira and R.M. Stephan, Analysis of passive magnetic bearings for kinetic energy storage systems, Linz, Austria, August 2014.

[13] E. Rodriguez, G.G. Sotelo, J.G De Oliveira, J. de Santiago, M. Rossander and R.M. Stephan, Designing, simulations and experiments of a passive permanent magnet bearing, International Journal of Applied Electromagnetics and Mechanics 51(2) (January 2016), 131-149.

[14] J. de Santiago and H. Bernhoff, Calculation of Tooth Ripple Losses in Solid Poles. Electric Power Components and Systems 43(3) (February 2015), 245-251.

[15] R.L. Stoll, The analysis of eddy currents, Clarendon Press, 1974.

[16] M. Talaat and N.H. Mostafa, Use of finite element method for the numerical analysis of eddy current brake, In 2014 15th International Workshop on Research and Education in Mechatronics (REM), September 2014, 1-7.

[17] R.K. Wangsness, Electromagnetic fields, Wiley, July 1986.

[18] J.-P. Yonnet, G. Lemarquand, S. Hemmerlin and E. Olivier-Rulliere, Stacked structures of passive magnetic bearings, Journal of Applied Physics 70(10) (1991), 6633-6635.

[19] P. Zhang, G.Y. Sizov, J. He, D.M. Ionel and N.A.O. Demerdash, Calculation of Magnet Losses in Concentrated-Winding Permanent-Magnet Synchronous Machines Using a Computationally Efficient Finite-Element Method, IEEE Transactions on Industry Applications 49(6) (November 2013), 2524-2532.

[20] G.-H. Zhou, L. Han, Z.-N. Fan, Y. Liao and S. Huang, 3d loss and heat analysis at the end region of 4-poles $1150 \mathrm{MW}$ nuclear power turbine generator, Archives of Electrical Engineering 63(1) (2014), 47-61.

[21] Z.Q. Zhu and D. Howe, Halbach permanent magnet machines and applications: A review, Electric Power Applications, IEE Proceedings 148(4) (2001), 299-308. 